\title{
BMJ Open Evaluation of the diagnostic accuracy of fractional exhaled nitric oxide (FeNO) in patients with suspected asthma: study protocol for a prospective diagnostic study
}

Christina Kellerer (1) , ${ }^{1}$ Alexander Hapfelmeier, ${ }^{1,2}$ Rudolf A Jörres, ${ }^{3}$ Konrad Schultz, ${ }^{4}$ Benjamin Brunn, ${ }^{1}$ Antonius Schneider ${ }^{1}$

To cite: Kellerer C,

Hapfelmeier A, Jörres RA, et al. Evaluation of the diagnostic accuracy of fractional exhaled nitric oxide (FeNO) in patients with suspected asthma: study protocol for a prospective diagnostic study. BMJ Open 2021;11:e045420. doi:10.1136/ bmjopen-2020-045420

- Prepublication history for this paper is available online. To view these files, please visit the journal online (http://dx.doi. org/10.1136/bmjopen-2020045420).

Received 30 September 2020 Revised 28 January 2021 Accepted 03 February 2021

Check for updates

(c) Author(s) (or their employer(s)) 2021. Re-use permitted under CC BY-NC. No commercial re-use. See rights and permissions. Published by BMJ.

For numbered affiliations see end of article.

Correspondence to

Christina Kellerer;

christina.kellerer@mri.tum.de

\section{ABSTRACT}

Introduction The measurement of fractional exhaled nitric oxide (FeNO) is promising for diagnosing asthma and might substitute for bronchial provocation (BP) tests. To evaluate the diagnostic accuracy of FeNO within a confirmatory study, the following hypotheses will be tested: (1) A FeNO cut-off $>50 \mathrm{ppb}$ (parts per billion) is suitable for diagnosing asthma (sensitivity $35 \%$, specificity $95 \%$ ); (2) If the clinical symptoms 'allergic rhinitis' and 'wheezing' are present, asthma can be diagnosed at FeNO >33 ppb with a positive predictive value (PPV) $\geq 70 \%$ and (3) A FeNO $>33$ ppb can predict responsiveness to inhaled corticosteroid (ICS) with a PPV $\geq 70 \%$.

Methods and analysis A prospective diagnostic study will be conducted in three practices of pneumologists in Germany. 300 patients suspected of suffering from asthma will be included. As an index test, patients perform FeNO measurement with the device NIOX VERO. As reference a test, patients are examined with whole bodyplethysmography and BP, if necessary. After 3 months, patients with an asthma diagnosis will be examined again to verify the diagnosis and evaluate ICS responsiveness. Patients who did not receive an asthma diagnosis at the initial examination will be phoned after 3 months and asked about persistent respiratory symptoms to exclude false negative findings. As a primary target, sensitivity and specificity of FeNO $>50 \mathrm{ppb}$ will be determined. As a secondary target the PPV for asthma at FeNO $>33$ ppb, when the symptoms 'allergic rhinitis' and 'wheezing' are present, will be calculated. Regarding ICS responsiveness, the PPV of FeNO > 33 ppb will be determined. Ethics and dissemination The study was approved by the Ethical Committee of the Technical University of Munich (Reference number 122/20 S). The major results will be published in peer-reviewed academic journals and disseminated through conferences.

Trial registration number DRKS00021125.

\section{INTRODUCTION}

\section{Background}

The diagnosis of asthma is limited by the fact that airway obstruction is often not present during investigation by spirometry or whole body plethysmography (WBP) when patients suffer from mild symptoms, thus leading to diagnostic
Strengths and limitations of this study

- As this prospective confirmatory study aims to validate predefined fractional exhaled nitric oxide (FeNO) cut-off values for an asthma diagnosis and inhaled corticosteroid responsiveness it might be able to determine the appropriate place of FeNO in the diagnosis of asthma and in routine care.

- A high-quality reference standard will be used in this study as the diagnosis of asthma will be made in all patients based on bronchial provocation tests assessed in whole body plethysmography and a potential asthma diagnosis will be verified after 3 months.

- Different devices might lead to different cut-off values. However, we are not able to compare FeNO devices from various manufacturers within this study.

- The present study is not able to assess the impact of FeNO on patient management in routine care because pneumologists will be blinded against FeNO values.

uncertainty. For these cases, diagnostic guidelines recommend bronchial provocation (BP) tests, which can only be performed in pneumologic centres in order to diagnose or exclude asthma. ${ }^{2}$ Moreover, peak-flow variability can be assessed, but the low diagnostic value of this method has been demonstrated and it is considered as a second choice method. ${ }^{34}$ Thus, in the case of inconclusive lung function results, BP remains the reference standard for the diagnosis of asthma. ${ }^{12}$

Numerous studies have demonstrated that, in addition to BP, the measurement of fractional exhaled nitric oxide (FeNO) has a high potential for diagnosing asthma and could possibly replace $\mathrm{BP}^{5}{ }^{6} \mathrm{NO}$ is released during type 2 allergic inflammation ${ }^{7}$ and it could be shown that patients with asthma, even in mild stages of the disease, exhale NO in higher concentrations. ${ }^{8}$ 
In contrast to $\mathrm{BP}, \mathrm{FeNO}$ is a non-invasive measurement that can be performed without risk to the patient in a short time.

The available studies indicate that a cut-off value of $50 \mathrm{ppb}$ (parts per billion) is well suited for diagnosing asthma. ${ }^{910}$ However, such values were identified only by post hoc analyses in the sense of multiple and exploratory testing. Accordingly, the major criticism is that the diagnostic value of the cut-off points identified and proposed so far need to be confirmed in a prospective study. ${ }^{19}$

It was shown in a secondary analysis that even lower FeNO values than $50 \mathrm{ppb}$ could be useful for diagnosis when considering appropriate anamnestic information. If, for example, the patient suffers from allergic rhinitis and wheezing, an asthma diagnosis can be established with a high degree of certainty when FeNO is $>33$ ppb. ${ }^{11}$ However, this algorithm needs to be validated in a multicentre study. Studies also indicate that the diagnostic accuracy of FeNO measurement might be superior to $\mathrm{BP}^{911}$, as the latter gives correctly positive values in only about $70 \%$ of cases. ${ }^{12}$ This might be especially true for allergic, inflammatory alterations of respiratory tract, which might be better diagnosed via FeNO than $\mathrm{BP} .{ }^{13}$ In line with this, FeNO could be suitable for predicting responsiveness to inhaled corticosteroide (ICS) in asthma. The study by Martin et $a l^{14}$ showed that FeNO $>33$ ppb could be used to predict the response to ICS in patients with suspected asthma with a high degree of certainty. However, these values were also identified by post hoc analyses. Another study found FeNO values $>40 \mathrm{ppb}$ to predict ICS responsiveness in patients with non-specific respiratory symptoms. ${ }^{15}$ In view of these reports, it is obvious that a prospective confirmatory study is necessary to validate predefined cut-off values and to determine the appropriate place of $\mathrm{FeNO}$ in the diagnosis of asthma as well as in routine care.

\section{Aims of the study}

The present study aims to evaluate sensitivity, specificity, positive predictive value (PPV) and negative predictive value (NPV) to clarify the following hypotheses:

1. Primary hypothesis: The sensitivity of FeNO measurement for diagnosing asthma is $35 \%$ at the cut-off $>50$ ppb, and specificity is $95 \%$.

2. Secondary hypothesis: if the clinical symptoms 'allergic rhinitis' and 'wheezing' are present, the PPV of FeNO $>33 \mathrm{ppb}$ is at least $70 \%$ (validation of the diagnostic algorithm $^{10}$ ). Sensitivity, specificity and NPV will also be estimated.

3. Further secondary hypothesis: The PPV of FeNO >33 ppb for ICS responsiveness is at least 70\%. Sensitivity, specificity and NPV will also be estimated.

\section{METHODS AND ANALYSIS \\ Trial design}

The study will be conducted as a multicentre diagnostic study in three practices of pneumologists. Patients with suspected asthma visiting one of the three practices will be informed about the study. After having obtained the informed consent of the patient, FeNO measurement will be carried out.
Afterwards, the patient will be routinely examined with WBP and, if necessary, BP to clarify a potential asthma diagnosis. This diagnostic procedure is routinely performed in German practices of pneumologists in ambulatory care if asthma is suspected.

Three months after inclusion, patients who have been diagnosed as suffering from asthma will be invited into the practices for a follow-up examination. Patients will perform FeNO measurement and afterwards they will be again examined with WBP and BP (when appropriate). Based on the examination with WBP and $\mathrm{BP}$ (when appropriate), it will be verified whether the patient has responded positively to ICS (delayed type of diagnostic study ${ }^{16}$ ). Based on the recommendations of the national ${ }^{1}$ and international ${ }^{2}$ asthma guidelines, this time interval is reasonable for a therapy of at least 3 months after initial diagnosis. ${ }^{12}$ Patients who did not receive an asthma diagnosis will be phoned after 3 months and asked whether the respiratory symptoms still persist in order to rule out false negative findings. Patients with persistent symptoms will be invited back for re-evaluation.

\section{Study setting}

The study will be conducted in three private practices of pneumologists in Germany ('Zentrum für Pneumologie, Onkologie und Schlafmedizin am Diakonissenkrankenhaus' in Augsburg; 'Lungenpraxis Starnberg' in Starnberg; 'Pneumologie Elisenhof' in Munich). Further practices will be included if necessary for sufficient recruitment within the intended time frame.

\section{Eligibility criteria}

\section{Inclusion criteria}

All adult patients suspected of suffering from asthma, who visit one of the three participating practices of pneumologists and declare their written informed consent to participate in the study will be included consecutively. Patients will be included regardless of the severity of respiratory symptoms.

\section{Exclusion criteria}

Patients with the following criteria are excluded:

- Patients who do not agree to participate in this study.

- Patients younger than 18 years (legal grounds).

- Patients who do not understand the meaning of the study due to a lack of knowledge of the German language.

- Patients with already diagnosed obstructive airway disease.

- Patients who smoked on the day of the examination (distortion of the FeNO results and reactivity during BP testing).

- Nitrate-rich meal (eg, salad) before the examination (false high FeNO values).

- Patients with respiratory infection $<6$ weeks before examination (distortion of the FeNO results and/or $\mathrm{BP})$.

\section{Recruitment and taking informed consent}

Patients visiting one of the three participating practices of pneumologists will be contacted by a doctoral candidate 


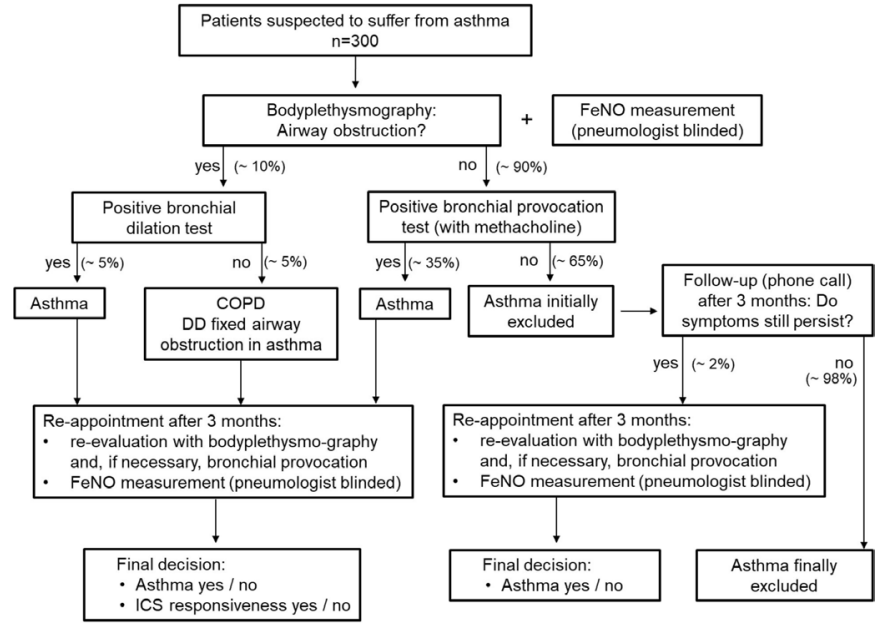

Figure 1 Overview of the diagnostic procedure during the conduct of the study. COPD, chronic obstructive pulmonary disease; DD, differential diagnosis; FeNO, fractional exhaled nitric oxide; ICS, inhaled corticosteroid.

(BB) from the Medical Faculty of the Technical University of Munich or a Research Associate at the Institute of General Practice and Health Services Research of the Technical University of Munich regarding possible participation in the study. They will check the inclusion and exclusion criteria and will inform the patient about the study. Finally, the attending pneumologist will provide detailed information about the study. In the case of patients who are screened for participation but who ultimately do not participate in the study (due to disagreement or other reasons), age, gender and the reason for non-participation will be documented anonymously in order to be able to conduct a non-responder analysis to assess a potential recruitment bias.

\section{Interventions}

Patients included in the study will be examined at first contact (time point $\mathrm{t} 1$ ) and 3 months later (time point $\mathrm{t} 2$ ). The diagnostic workup is summarised in figure 1 and the patient timeline in figure 2.

\section{Initial examination (T1)}

During the first presentation for diagnostic workup in one of the participating practices of pneumologists, patients are examined with a NO-measuring device (NIOX VERO) as an index test. Afterwards patients will be examined with WBP as a reference standard; a BP test is performed additionally as part of the diagnostic routine, if required by the pneumologist. In addition, patients complete a questionnaire with structured questions about medical history and symptoms. The questionnaire also contains the 'Asthma Control Questionnaire (ACQ)'. ${ }^{17}$ The ACQ is used to determine the extent of asthma control (controlled, partially controlled, uncontrolled) and the responsiveness to ICS. ICS responsiveness is given if the ACQ score improves by at least 0.5 in the sense of a 'minimal important difference'. ${ }^{14} 18$ Type and daily dose of ICS are recorded.

\begin{tabular}{|c|c|c|c|c|}
\hline & \multicolumn{4}{|c|}{ STUDY PERIOD } \\
\hline \multirow{3}{*}{ TIMEPOINT } & Enrolment & $\begin{array}{c}\text { Assessments and } \\
\text { interventions }\end{array}$ & Follow-up (3 n & onths after t1) \\
\hline & $t_{0}$ & $t_{1}$ & \multicolumn{2}{|c|}{$t_{2}$} \\
\hline & & & $\begin{array}{c}\text { Asthma } \\
\text { diagnosis at t1 }\end{array}$ & $\begin{array}{c}\text { No asthma } \\
\text { diagnosis at t1 }\end{array}$ \\
\hline \multicolumn{5}{|l|}{ ENROLMENT: } \\
\hline \multirow{2}{*}{$\begin{array}{l}\text { Eligibility screen } \\
\text { Informed consent }\end{array}$} & $x$ & & & \\
\hline & $x$ & & & \\
\hline \multicolumn{5}{|l|}{ INTERVENTIONS: } \\
\hline \multirow{2}{*}{$\begin{array}{r}\text { Index test: FeNO } \\
\text { Reference test: } \\
\text { Bodyplethysmography } \\
\text { and bronchial } \\
\text { provocation test } t^{*}\end{array}$} & & $x$ & $x$ & \\
\hline & & $x$ & $x$ & \\
\hline \multicolumn{5}{|l|}{ ASSESSMENTS: } \\
\hline$A C Q$ & & $\mathrm{x}$ & $\mathrm{x}$ & \\
\hline $\begin{array}{r}\text { Self-reported } \\
\text { questionnaire }\end{array}$ & & $x$ & $x$ & \\
\hline $\begin{array}{r}\text { Structured interview } \\
\text { (phone call) }\end{array}$ & & & & $\mathrm{x}$ \\
\hline
\end{tabular}

Figure 2 Standard Protocol Items: Recommendations for interventional trials schedule. ${ }^{*}$ Bronchial provocation test is only performed if required by the pneumologist. ACQ, Asthma Control Questionnaire; FeNO, fractional exhaled nitric oxide.

\section{Index test}

The index test is performed with the electrochemically based NO-measuring device NIOX VERO. This device is CE certified, available in national and international markets, and is already widely used in practices. The FeNO measurements are performed once for each patient according to the recommendations of the ATS (American Thoracic Society) and ERS (European Respiratory Society). ${ }^{19}$ It is a non-invasive measurement since the patient only needs to take a deep breath through the device and exhale evenly. The FeNO measurements are performed by a doctoral candidate, or a research assistant, or a lung function assistant according to the instructions of the manufacturer. FeNO devices and measurements are provided by the Institute of General Practice and Health Services Research of the Technical University of Munich.

\section{Reference test}

Following the FeNO measurement, an examination with WBP is routinely performed and, if required by the pneumologist, $\mathrm{BP}$ is performed as part of the diagnostic routine to rule-in or rule-out the diagnosis of asthma. In Germany, both of these assessments are routine tests and would also take place outside the study. Thus, there is no funding of these measurements.

\section{Whole body plethysmography}

WBP is considered as the reference standard used to diagnose obstructive airway diseases. An obstructive airway disease is indicated if forced expiratory volume in $1 \mathrm{~s}\left(\mathrm{FEV}_{1}\right)$ and/or $\mathrm{FEV}_{1} / \mathrm{FVC}$ are below their lower limits of normal. ${ }^{20} \mathrm{~A}$ reversible airway obstruction is diagnosed if the bronchodilation test is positive $\left(\Delta \mathrm{FEV}_{1}>12 \%\right.$ and $200 \mathrm{~mL}$ ). If there is no bronchial obstruction, $\mathrm{BP}$ is performed. 


\section{BP test}

$\mathrm{BP}$ is performed to determine bronchial hyperresponsiveness (BHR) to methacholine according to the 1-concentration-4-step dosimeter protocol. ${ }^{21}$ This yields similar results as the ATS multiconcentration protocol ${ }^{22}$ but offers advantages in clinical practice. The test is considered positive (indicating BHR) if $\mathrm{FEV}_{1}$ decreases by at least $20 \%$ after inhalation of a maximum cumulative methacholine dose of $960 \mu \mathrm{g}$, and/or if specific airway resistance (sRaw) increases simultaneously by at least $100 \%$ and to at least $2.0 \mathrm{kPa} * \mathrm{~s}$, and/or if airway resistance (Raw) increases simultaneously by at least $100 \%$ and to at least $0.5 \mathrm{kPa}^{*} \mathrm{~s} / \mathrm{L}^{22}{ }^{23}$

\section{Follow-up examination after 3 months (T2)}

A single BP test as a reference standard for the diagnosis of asthma only reflects the situation at the time of examination. In some cases, patients with a positive BP test do not suffer from asthma (false positive), since the PPV of BP is only about $70 \% .^{12}{ }^{24}$ According to the German guideline 'NVL Asthma' and international guideline GINA, ${ }^{12}$ a minimum of 3 months of therapy with ICS is recommended at the time of initial diagnosis (maintenance therapy) before a dose reduction can be started (stepping down). Accordingly, after 3 months, all patients with a positive BP test or with the diagnosis of asthma, respectively, will be asked to return to the practice. During this follow-up, appointment patients will receive an FeNO measurement and afterwards they will be examined with WBP. If asthma has been diagnosed at $\mathrm{t} 1$ based on BP, BP will be repeated at $\mathrm{t} 2$ (if the result of the bodyplethysmographic examination is inconspicuous). ICS responsiveness is diagnosed when an airway obstruction is reversible or the tolerance to BP increases by at least one level ('doubling dose'). In addition, a potential improvement in respiratory symptoms is assessed by the ACQ. Potential changes in FeNO values between $\mathrm{t} 1$ and $\mathrm{t} 2$ will be evaluated exploratory.

Approximately $2 \%$ of patients can be diagnosed with false negatives by BP tests (NPV of BP determined in WBP: $98 \%{ }^{12}$ ). Therefore, patients with an inconspicuous BP test are phoned after 3 months in order to rule out a false negative test result. Patients will be interviewed regarding symptoms and inhaler medication (structured telephone interview). An interview will take about $5 \mathrm{~min}$. If patients report persistent respiratory symptoms although the BP test was negative, they will be offered a follow-up examination at the practice of the respective pneumologist. Depending on the findings, another BP test assessed by WBP will be performed. This will be decided by the pneumologist in each individual case.

\section{Diagnostic decision making}

A committee of experts (Antonius Schneider, member of the author board of the NVL Asthma; Rudolf A. Jörres, Senior Scientist for Respiratory Diseases, Occupational Medicine, LMU (Ludwig-Maximilians Universität, Munich); Konrad Schultz, Medical Director of the
Rehabilitation Clinic for Pneumology Bad Reichenhall) will review each diagnosis in consideration of the patient's medical history, WBP investigation and BP. The respective pneumologists will be contacted in each inconsistent case to clarify the diagnosis. In addition, the committee of experts assesses whether the patients responded to ICS (delayed type of diagnostic study ${ }^{16}$ ).

At least one criterion must be given for an asthma diagnosis at $\mathrm{t} 1$ :

1. Increase of $\mathrm{FEV}_{1}$ from baseline by $>12 \%$ and by $>200$ $\mathrm{mL}$ during bronchodilation testing if airway obstruction exists (NVL Asthma ${ }^{1}$ ).

2. Positive response of $\mathrm{FEV}_{1}$ or Raw or sRaw during $\mathrm{BP}$ test.

At least one of the following criteria at $\mathrm{t} 2$ must be fulfilled to establish ICS responsiveness at t2:

1. Increase of $\mathrm{FEV}_{1}$ from baseline (t1) by $>12 \%$ and by $>200 \mathrm{~mL}\left(\mathrm{NVL}\right.$ asthma $\left.{ }^{1}\right)$.

2. Increase of tolerance during BP tests by at least one level.

3. Improvement by 0.5 score points in the ACQ.

If criterion 1 is fulfilled, a BP test is not performed. In addition, it is not performed if the patient reports a worsening of respiratory symptoms since the initial presentation at $\mathrm{t} 1$.

\section{Blinding}

The FeNO measurements are performed by a doctoral candidate, a research assistant or a lung function assistant and are documented on a structured sheet. The pneumologist who assesses the results of WBP and BP tests, is blinded to the results of the FeNO measurement. The results of the examinations and the diagnosis made by the pneumologist are documented on a separate sheet.

The committee of experts (who finally diagnoses or excludes asthma in each individual case and assesses whether the patient responded to ICS) is also blinded to the results of FeNO measurement. The committee only has access to the results of bodyplethysmographic measurements, BP tests and anamnestic information.

\section{Data management and monitoring}

Immediately after signing the patient information, consent and data protection declaration, a pseudonymised study ID (identification number) is assigned to the patient, under which the further data and study results are documented and stored. From now, all other personal data and findings will only be passed on in encrypted form, that is, neither the name nor the initials nor the exact date of birth will appear in the encryption code. The patient identification list remains at the Institute of General Practice and Health Services Research and is only accessible to authorised study personnel. The doctoral candidate enters all data from the patient's files, the values of the FeNO measurement, and the values of the lung function tests obtained by WBP in encrypted form into the statistical program SPSS (Version 26, IBM Corp., Armonk, NY, USA). Five per cent of the data will be 
entered twice to estimate the frequency of typing errors. Moreover, all FeNO values and all asthma diagnoses are entered twice to allow a complete correction of possible typing errors. If no more corrections are required in the database, it is closed and will be used for statistical evaluation. The data collection process and the study procedures will be supervised by a research associate who will also perform periodic visits to the practices.

\section{Statistics}

Sample size estimation

According to previous studies in practices of pneumologists, a sample size of $n=300$ can be expected to include about 105 patients with a new diagnosis of asthma. The prevalence in a previous study in a large lung specialist practice was $39 \% .^{5}$ To be on the safe side, we assume a slightly lower prevalence of $35 \%$ for the current multicentre study. The two primary endpoints will be tested confirmatory on two-sided 5\% significance levels. A hierarchical test procedure is used to control the global type 1 error at a $5 \%$ significance level. Using exact binomial tests, the expected specificity of $95 \%$ is first tested against a reference value of $90 \%$ assumed under the null hypothesis. If the test result is positive, another confirmatory test of the expected sensitivity of $35 \%$ against a reference value of $20 \%$ will follow. These tests each achieve a power of $90 \%$ with a sample sizes of 195 patients without asthma diagnosis and 105 asthma patients. ${ }^{25}$ The total number of patients is therefore 300 .

A validation of the diagnostic algorithm (FeNO, 'Allergic Rhinitis' and 'Wheezing') ${ }^{10}$ is performed by means of Wilcoxon (Mann-Whitney) rank sum tests. With the sample sizes mentioned above, this test reaches a power of $80 \%$ at a two-sided and exploratory $5 \%$ significance level to detect a diagnostic accuracy of area under the curve $(\mathrm{AUC})=0.60 .^{26}$

\section{Statistical analysis}

Patients participating in the study are characterised by descriptive statistics (mean values, SD, medians, minimum, maximum; absolute and relative frequencies).

As primary and confirmatory analysis, exact binomial tests of sensitivity and specificity at $\mathrm{t} 1$ are performed hierarchically at the predetermined FeNO cut-off value of $>50 \mathrm{ppb}$, each against a reference value of $90 \%$ or $20 \%$, respectively, and at the two-sided 5\% significance level. For these measures as well as for PPV and NPV, corresponding 95\% CIs are calculated. Fagan nomograms will be provided for the PPV and NPV to enable the exploration of post-test probabilities depending on the population specific prevalence. The distribution of diagnoses using $\mathrm{FeNO}$ and the reference standard will be shown in a cross-table. The reference standard is the diagnosis of asthma made by body plethysmography and bronchoprovocation if necessary. The statistics mentioned above are calculated analogously:

- In the presence of the symptoms 'Allergic rhinitis' and 'Wheezing' and using an FeNO cut-off value of
$>33 \mathrm{ppb}$ at $\mathrm{t} 1$. In addition, the AUC of the receiver operating characteristic curve is determined with a corresponding $95 \% \mathrm{CI}$ and tested against a reference value of 0.50 using the Wilcoxon (Mann-Whitney) rank sum test.

- For the prediction of ICS responsiveness (determined at t2) using an FeNO cut-off value of $>33$ ppb.

In accordance with the secondary hypotheses, exploratory testing of the PPV values will be performed by exact binomial tests on two-sided $5 \%$ significance levels against a reference value of $70 \%$.

Changes in the ACQ during follow-up will be estimated by secondary analyses. For this purpose, a composite endpoint related to ICS responsiveness will be developed (at least one out of these criteria must be fulfilled):

1. Increase of $\mathrm{FEV}_{1}$ from baseline (t1) by $>12 \%$ and by $>200 \mathrm{~mL}\left(\mathrm{NVL}\right.$ asthma $\left.{ }^{1}\right)$.

2. Increase of tolerance during BP tests by one level.

3. Improvement of 0.5 score points in the ACQ.

Moreover, regarding ICS responsiveness potential changes in FeNO values between the first appointment (t1) and the follow-up appointment (t2) will be evaluated exploratory in secondary analyses. Additionally, subgroup analyses related to different cut-off values of BP will be performed. Furthermore, the influence of anthropometric parameters on $\mathrm{FeNO}$ values will be analysed in secondary analyses.

\section{Patient and public involvement}

Patients were not involved in the design of this study.

\section{DISCUSSION}

The present confirmatory diagnostic study aims to prove the diagnostic benefit of FeNO measurement regarding the diagnosis of asthma. FeNO is an attractive diagnostic tool and provides a non-invasive marker of inflammatory processes in the lung. ${ }^{8}$ In contrast, $\mathrm{BP}$ as the reference standard for diagnosing asthma is time-consuming, costintensive, often only available in specific lung function laboratories and bears a small risk of bronchospasm. ${ }^{22}$ Therefore, it is reasonable to discuss FeNO measurement as an alternative procedure to diagnose asthma. Beyond that, there are strong hints that it has added value to determine ICS-responsiveness. Accordingly, a health technology assessment found that the inclusion of FeNO measurement into the diagnostic pathway might increase the diagnostic cost-effectiveness. ${ }^{27}$

Several studies have already shown a high diagnostic accuracy of $\mathrm{FeNO}$ for discerning asthma in patients suspected of suffering from asthma. ${ }^{56}$ In most of these studies, values of specificity were superior to those of sensitivity, suggesting that FeNO measurement is more suitable for ruling in than for ruling out the disease. ${ }^{9}$ However, a great weakness of the studies published so far is that the optimal FeNO cut-off values were defined post hoc. This probably led to differences when estimating the diagnostic accuracy of FeNO in different studies as well as 
to discrepancies regarding the optimal cut-off value for diagnosing or excluding asthma. Indeed, it is known that diagnostic algorithms, including cut-off values perform better in the dataset from which they are derived, compared with a dataset with even similar but different individuals. ${ }^{28}$ This phenomenon can be explained, among other factors, by overfitting, the absence of important predictors, unsatisfactory model derivation and differences between patient samples. ${ }^{29}{ }^{30}$ It is, therefore, essential to validate predefined FeNO cut-off values and diagnostic algorithms based on FeNO measurements in a prospective study, for example, in individuals outside the derivation dataset, in order to be able to determine the adequate place of $\mathrm{FeNO}$ measurement in the diagnosis of asthma and in routine care. ${ }^{19}$ The present confirmatory study aims to close this gap.

Due to the confirmatory character of this study, three hypotheses are proposed before the study is conducted. First, we hypothesise that an FeNO cut-off value of $>50$ $\mathrm{ppb}$ is suitable to diagnose asthma (sensitivity $35 \%$, specificity $95 \%$ ). Second, we test the validity of the assumption that asthma can be diagnosed with a certainty (PPV) of at least $70 \%$ at an FeNO value of $>33 \mathrm{ppb}$, if the clinical symptoms 'allergic rhinitis' and 'wheezing' are present. Moreover, in line with the study by Martin et $a l^{14}$ we hypothesise that an $\mathrm{FeNO}$ value of $>33 \mathrm{ppb}$ can predict an ICS responsiveness with a certainty (PPV) of at least $70 \%$. We are aware of the discussion about using FeNO measurement better to identify responsiveness to treatment rather than to label patients with a diagnosis. ${ }^{13} \mathrm{We}$ aim to investigate the diagnostic usefulness regarding these aspects in a confirmatory manner. Thus, the design of the study should be suitable to verify these hypotheses.

The study will be conducted prospectively by enrolling 300 diagnostic-naive patients from three different practices of pneumologists to increase the generalisability of the study. ${ }^{31}$ All patients will be subjected to the reference standard to establish their true diagnosis. In this context, a major strength of the study is that the diagnosis of asthma will be made rigorously on basis of BP in WBP. It has been shown previously that interpretation of $\mathrm{BP}$ responsiveness with WBP, including airway resistance, is superior to the interpretation solely based on $\mathrm{FEV}_{1}$ responsiveness. ${ }^{12}$ After 3 months, patients with an asthma diagnosis will be examined again and the diagnosis of asthma will be verified by the expert team in order to ensure the diagnosis, exclude false positive findings and determine ICS responsiveness. In parallel, patients without an asthma diagnosis will be phoned after 3 months and asked if respiratory symptoms still persist. Patients with persistent symptoms will be invited for re-evaluation to exclude false negative findings. This procedure enables us to determine the prognostic value of FeNO regarding the diagnosis of asthma, and to compare the diagnostic-prognostic value of FeNO with BP. The diagnosis of each patient, as well as the evaluation of ICS responsiveness, will be made by an expert team based on anamnestic information as well as on lung function measurements, including BP tests. The expert team as well as the pneumologists of the practices are blinded to the results of $\mathrm{FeNO}$ measurement to avoid information bias.

A limitation of the study might be that a longer course of disease could be taken into account, for example, with a 12-month follow-up evaluation. However, this would not allow us to use the optimal time frame of 3 months for determining ICS responsiveness. Moreover, another limitation of the study might be the fact that the presence of allergic rhinitis is reported by the patient without objective validation. However, this represents the typical state of knowledge in clinical practice as it is uncommon to verify the presence of allergic rhinitis with nasal provocation in pneumological practices. Moreover, it has to be mentioned that we could not include special measures to control for adherence regarding ICS inhalation and consequently this aspect cannot be controlled in this study. Beyond that FeNO devices from various manufacturers should be compared since it cannot be excluded that optimal cut-off values differ between devices. We think that determination of FeNO with NIOX VERO will allow a valid estimation, because it measures $\mathrm{FeNO}$ at a mouth flow rate of $50 \mathrm{~mL} / \mathrm{s}$ over $10 \mathrm{~s}$ and a pressure of 10 $\mathrm{cm} \mathrm{H}_{2} \mathrm{O}$ as per guideline recommendation, ${ }^{32}$ and NIOX has been used in many diagnostic studies. ${ }^{9}$ The present study might be able to enhance the implementation of FeNO in diagnostic guidelines. However, it will not be able to assess the impact of FeNO on diagnostic decision making in routine care and patient outcomes. This point can be only clarified in a clinical impact analysis study, which will be needed in future..$^{28334}$

\section{ETHICS AND DISSEMINATION}

The study was approved by the Ethical Committee of the Technical University of Munich (Reference number 122/20 S). Written, informed consent to participate will be obtained from all participants. The major results of the study will be published in peer-reviewed academic journals and disseminated through conferences.

\section{TRIAL STATUS}

Protocol V.1.0. For recruitment the following time frame is planned: First patient in July 2020, last patient in September 2021, last patient out December 2021.

\section{Author affiliations \\ ${ }^{1}$ Technical University of Munich, School of Medicine, Institute of General Practice and Health Services Research, Munich, Germany \\ ${ }^{2}$ Institute of Medical Informatics, Statistics and Epidemiology, School of Medicine, Technical University of Munich, Munich, Germany \\ ${ }^{3}$ Institute and Outpatient Clinic for Occupational, Social and Environmental Medicine, Ludwig-Maximilians-Universität München, Munich, Germany \\ ${ }^{4}$ Clinic Bad Reichenhall, Center for Rehabilitation, Pneumology and Orthopedics, Bad Reichenhall, Germany}

Acknowledgements We are grateful to Drs Hellmann, Wehgartner-Winkler, Faderl, Dankelmann, Vitiello and Schlimok in Augsburg, to Dr Weber in Starnberg, and to Dr Powitz in Munich for the opportunity to perform the study in their practices. We also 
thank the technical personnel for making the study possible through logistic and organisational support. In addition, we appreciate the willingness of all participants to perform the additional FeNO measurements.

Contributors CK prepared the final study protocol, was involved in the development of the design of the study and agreed to be accountable for all aspects of the work. AH developed the details of the statistical analysis plan, reviewed the manuscript and commented on drafts of the final manuscript. RAJ helped with the development of the design of the study and with manuscript preparation. KS and BB contributed to the development of the study protocol and helped with writing. AS developed the design of the study and was involved in the development of the statistical analysis plan as well as in manuscript preparation.

Funding The authors have not declared a specific grant for this research from any funding agency in the public, commercial or not-for-profit sectors.

\section{Competing interests None declared.}

Patient and public involvement Patients and/or the public were not involved in the design, or conduct, or reporting, or dissemination plans of this research.

Patient consent for publication Not required.

Provenance and peer review Not commissioned; externally peer reviewed.

Open access This is an open access article distributed in accordance with the Creative Commons Attribution Non Commercial (CC BY-NC 4.0) license, which permits others to distribute, remix, adapt, build upon this work non-commercially, and license their derivative works on different terms, provided the original work is properly cited, appropriate credit is given, any changes made indicated, and the use is non-commercial. See: http://creativecommons.org/licenses/by-nc/4.0/.

ORCID iD

Christina Kellerer http://orcid.org/0000-0002-0670-6392

\section{REFERENCES}

1 Bundesärztekammer (BÄK), Kassenärztliche Bundesvereinigung (KBV), Arbeitsgemeinschaft Der Wissenschaftli-chen Medizinischen Fachgesellschaften (AWMF). Nationale VersorgungsLeitlinie asthma - Langfassung, 4. Auflage. Konsultationsfassung., 2020. Available: www.asthma.versorgungsleitlinien.de [Accessed 22 Jun 2020].

2 Global Initiative for Asthma. Global strategy for asthma management and prevention, 2020. Available: www.ginasthma.org

3 Künzli N, Stutz EZ, Perruchoud AP, et al. Peak flow variability in the SAPALDIA study and its validity in screening for asthmarelated conditions. The SPALDIA team. Am J Respir Crit Care Med 1999;160:427-34.

4 Tilemann L, Gindner L, Meyer F, et al. Diagnostischer Wert Der Peak-Flow-Variabilität bei Verdacht auf asthma bronchiale in Der Hausarztpraxis. Dtsch med Wochenschr 2009;134:2053-8.

5 Schneider A, Schwarzbach J, Faderl B, et al. FENO measurement and sputum analysis for diagnosing asthma in clinical practice. Respir Med 2013;107:209-16.

6 Schneider A, Faderl B, Schwarzbach J, et al. Prognostic value of bronchial provocation and FENO measurement for asthma diagnosis--results of a delayed type of diagnostic study. Respir Med 2014;108:34-40.

7 Bjermer L, Alving K, Diamant Z, et al. Current evidence and future research needs for FeNO measurement in respiratory diseases. Respir Med 2014;108:830-41.

8 Lane C, Knight D, Burgess S, et al. Epithelial inducible nitric oxide synthase activity is the major determinant of nitric oxide concentration in exhaled breath. Thorax 2004;59:757-60.

9 Karrasch S, Linde K, Rücker G, et al. Accuracy of FENO for diagnosing asthma: a systematic review. Thorax 2017;72:109-16.

10 Schneider A, Linde K, Reitsma JB, et al. A novel statistical model for analyzing data of a systematic review generates optimal cutoff values for fractional exhaled nitric oxide for asthma diagnosis. $J$ Clin Epidemiol 2017;92:69-78.

11 Schneider A, Wagenpfeil G, Jörres RA, et al. Influence of the practice setting on diagnostic prediction rules using FENO measurement in combination with clinical signs and symptoms of asthma. BMJ Open 2015;5:e009676.

12 Schneider A, Schwarzbach J, Faderl B, et al. Whole-Body plethysmography in suspected asthma: a prospective study of its added diagnostic value in 302 patients. Dtsch Arztebl Int 2015;112:405-11.

13 Alving K. FeNO and suspected asthma: better to identify responsiveness to treatment than to label with a diagnosis. Lancet Respir Med 2018;6:3-5.

14 Martin MJ, Wilson E, Gerrard-Tarpey W, et al. The utility of exhaled nitric oxide in patients with suspected asthma. Thorax 2016;71:562-4.

15 Price DB, Buhl R, Chan A, et al. Fractional exhaled nitric oxide as a predictor of response to inhaled corticosteroids in patients with non-specific respiratory symptoms and insignificant bronchodilator reversibility: a randomised controlled trial. Lancet Respir Med 2018;6:29-39.

16 Knottnerus JA, Muris JW. Assessment of the accuracy of diagnostic tests: the cross-sectional study. J Clin Epidemiol 2003;56:1118-28.

17 Juniper EF, O'Byrne PM, Guyatt GH, et al. Development and validation of a questionnaire to measure asthma control. Eur Respir $J$ 1999;14:902-7.

18 Juniper EF, Svensson K, Mörk A-C, et al. Measurement properties and interpretation of three shortened versions of the asthma control questionnaire. Respir Med 2005;99:553-8.

19 , American Thoracic Society, European Respiratory Society. ATS/ ERS recommendations for standardized procedures for the online and offline measurement of exhaled lower respiratory nitric oxide and nasal nitric oxide, 2005. Am J Respir Crit Care Med 2005;171:912-30.

20 Quanjer PH, Stanojevic S, Cole TJ, et al. Multi-ethnic reference values for spirometry for the 3-95-yr age range: the global lung function 2012 equations. Eur Respiratory Soc 2012.

21 Merget R, Jörres RA, Heinze E, et al. Development of a 1-concentration-4-step dosimeter protocol for methacholine testing. Respir Med 2009;103:607-13.

22 Crapo RO, Casaburi R, Coates AL, et al. Guidelines for methacholine and exercise challenge testing-1999. This official statement of the American thoracic Society was adopted by the ATS board of directors, July 1999. Am J Respir Crit Care Med 2000;161:309-29.

23 Criée CP, Sorichter S, Smith HJ, et al. Body plethysmography-its principles and clinical use. Respir Med 2011;105:959-71.

24 Perpiñá M, Pellicer C, de Diego A, et al. Diagnostic value of the bronchial provocation test with methacholine in asthma. A Bayesian analysis approach. Chest 1993;104:149-54.

25 Chow S-C, Wang H, Shao J. Sample size calculations in clinical research. CRC press, 2007.

26 Noether GE. Sample size determination for some common nonparametric tests. J Am Stat Assoc 1987;82:645-7.

27 Harnan SE, Tappenden P, Essat M. Measurement of exhaled nitric oxide concentration in asthma: a systematic review and economic evaluation of NIOX MINO, NIOX Vero and NObreath. Health Technol Assess 2015;19:1-330.

28 Moons KGM, Kengne AP, Grobbee DE, et al. Risk prediction models: II. External validation, model updating, and impact assessment. Heart 2012;98:691-8.

29 Steyerberg EW. Clinical prediction models. Springer, 2019.

30 Toll DB, Janssen KJM, Vergouwe Y, et al. Validation, updating and impact of clinical prediction rules: a review. J Clin Epidemiol 2008;61:1085-94.

31 Justice AC, Covinsky KE, Berlin JA. Assessing the generalizability of prognostic information. Ann Intern Med 1999;130:515-24.

32 Dweik RA, Boggs PB, Erzurum SC, et al. An official ATS clinical practice guideline: interpretation of exhaled nitric oxide levels (FENO) for clinical applications. Am J Respir Crit Care Med 2011;184:602-15.

33 Moons KGM, Altman DG, Vergouwe Y, et al. Prognosis and prognostic research: application and impact of prognostic models in clinical practice. BMJ 2009;338:b606.

34 Cowley LE, Farewell DM, Maguire S, et al. Methodological standards for the development and evaluation of clinical prediction rules: a review of the literature. Diagn Progn Res 2019;3:16. 